\title{
Quantum Discontinuity for Massive Gravity with a Cosmological Term
}

\author{
Hisham Sati ${ }^{1}$ \\ Michigan Center for Theoretical Physics, \\ Randall Laboratory, Department of Physics, University of Michigan, \\ Ann Arbor, MI 48109-1120
}

\begin{abstract}
We report on the recent work on the van Dam-Veltman-Zakharov discontinuity for massive and partially massless gravitons in $\mathrm{A}(\mathrm{dS})$ space at one-loop.

The question of whether the graviton has a small non-zero mass or exactly zero mass has been addressed by van Dam and Veltman, and Zakharov[1]. The difference is physically detectible, e.g. by effect on bending of light by the sun. The case for non-zero $\Lambda$ has been studies by references [2] and [3] for de-Sitter and Anti-deSitter space respectively. In the latter it was found that the massive graviton has a smooth limit. An interesting phenomenon [4] that occurs in (A)dS space is partial masslessness with reduced degrees of freedom
\end{abstract}

\begin{tabular}{l|c|r}
\hline Theory & D.O.F. & Unitary? \\
\hline$M^{2}<\frac{2}{3} \Lambda$ & 5 & No \\
\hline$M^{2}=\frac{2}{3} \Lambda$ & 4 & Yes \\
\hline$M^{2}=0$ & 2 & Yes \\
\hline
\end{tabular}

All the above analysis is classical. One might ask the question what happens when loop effects are taken into account. It was shown [5] that the discontinuity persists in the flat space case. In [6] it was shown that this is also true in the case $\Lambda \neq 0$.

The starting point of the analysis is our starting point will be the action

$$
S\left[h_{\mu \nu}, T_{\mu \nu}\right]=S_{L}\left[h_{\mu \nu}\right]+S_{M}\left[h_{\mu \nu}\right]+S_{T}[h \cdot T]
$$

where $S_{L}$ the Einstein-Hilbert action $S_{E}=-\frac{1}{16 \pi G} \int_{M} \mathrm{~d}^{4} x \sqrt{\hat{g}}(\hat{R}-2 \Lambda)$, linearized about a background metric $g_{\mu \nu}$ according to $\hat{g}_{\mu \nu}=g_{\mu \nu}+\kappa h_{\mu \nu}$. If the background metric is Einstein, then the linearized action for $h_{\mu \nu}$ is

$$
S_{L}=\int \mathrm{d}^{4} x \sqrt{g}\left[\frac{1}{2} \tilde{h}^{\mu \nu}\left(-g_{\mu \rho} g_{\nu \sigma} \square-2 R_{\mu \rho \nu \sigma}\right) h^{\rho \sigma}-\nabla^{\rho} \tilde{h}_{\rho \mu} \nabla^{\sigma} \tilde{h}_{\sigma}^{\mu}\right]
$$

where $\tilde{h}_{\mu \nu}=h_{\mu \nu}-\frac{1}{2} g_{\mu \nu} h_{\sigma}^{\sigma}$. The linearized Lagrangian $S_{L}$ has a diffeomorphism symmetry which is broken when we add the Pauli-Fierz spin-2 mass term

$$
S_{M}=\frac{M^{2}}{2} \int \mathrm{d}^{4} x \sqrt{g}\left[h^{\mu \nu} h_{\mu \nu}-\left(h_{\mu}^{\mu}\right)^{2}\right]
$$

\footnotetext{
${ }^{1}$ hsati@umich.edu

CP624, Cosmology and Elementary Particle Physics, edited by B. N. Kursunoglu et al. (C) 2002 American Institute of Physics 0-7354-0073-3/02/\$19.00 
The source term for $T_{\mu \nu}$ (assumed conserved) is given by

$$
S_{T}=\int d^{4} x \sqrt{g} h_{\mu \nu} T^{\mu \nu}
$$

In order to neatly compare the massive (and partially massless) case with the massless one, we use the Stückelberg formalism to restore gauge symmetry. This is done by introducing an auxiliary vector field $V_{\mu}$ and then making a change of field integration variables $h_{\mu \nu} \rightarrow h_{\mu \nu}-\frac{2}{M} \nabla_{(\mu} V_{\nu)}$. The gauge invariances are then

\begin{tabular}{|l|c|}
\hline massive & partially massless \\
\hline$h_{\mu \nu} \rightarrow h_{\mu \nu}+2 \nabla_{(\mu} \xi_{\nu)}$ & $h_{\mu \nu} \rightarrow h_{\mu \nu}+2 \nabla_{(\mu} \xi_{\nu)}+\frac{2}{3} \Lambda g_{\mu \nu} \alpha$ \\
$V_{\mu} \rightarrow V_{\mu}+M \xi_{\mu}$ & $V_{\mu} \rightarrow V_{\mu}+M\left[\xi_{\mu}-\nabla_{\mu} \alpha\right]$ \\
\hline
\end{tabular}

Note that the parameters $\xi_{\mu}$ and $\alpha$ correspond to diffeomorphisms and Weyl rescalings respectively. For gauge-fixing we use a lagrangian quadratic in the constraint

$$
M V_{\mu}-\nabla^{\rho} \tilde{h}_{\rho \mu}=0 .
$$

with the additional constraint $h=0$ for the partially massless case. We decompose the metric fluctuation into traceless and scalar parts given respectively by $\phi_{\mu \nu} \equiv$ $h_{\mu \nu}-\frac{1}{4} g_{\mu \nu} h_{\sigma}^{\sigma}$, and $\phi \equiv h_{\sigma}^{\sigma}$ so that

$$
\begin{aligned}
h_{\mu \nu} & =\phi_{\mu \nu}+\frac{1}{4} g_{\mu \nu} \phi, \\
\tilde{h}_{\mu \nu} & =\phi_{\mu \nu}-\frac{1}{4} g_{\mu \nu} \phi, \\
h_{\mu}^{\mu} & =\phi
\end{aligned}
$$

The source will similarly be split into its irreducible components $T_{\mu \nu}=j_{\mu \nu}+\frac{1}{4} g_{\mu \nu} j$. The corresponding differential (Lichnerowicz)operators are given as

\begin{tabular}{l|c|l}
\hline spin & dimension & operator \\
\hline 2 & 9 & $\Delta(1,1) \phi_{\mu} \nu=-\square \phi+R_{\mu \tau} \phi_{\nu}^{\tau}+R_{\nu \tau} \phi_{\mu}^{\tau}-2 R_{\mu \rho \nu \tau} \phi^{\rho \tau}$ \\
\hline 1 & 4 & $\Delta\left(\frac{1}{2}, \frac{1}{2}\right) \xi_{\mu} \equiv-\square \xi_{\mu}+R_{\mu \nu} \xi^{\nu}$ \\
\hline 0 & 1 & $\Delta(0,0) \equiv-\square$ \\
\hline
\end{tabular}

At this stage one has to take into account the vector ghosts and the corresponding Faddeev-popov determinant. Now we restore yet another gauge invariance, namely that for the "massive" spin-1 field $V^{\mu}$. As before, we impose gauge symmetry by multiplying by a path-integral $\int \mathcal{D} \chi$ over a new decoupled scalar field $\chi$ and then making the change of variables $V_{\mu} \rightarrow V_{\mu}-M^{-1} \nabla_{\mu} \chi$. By construction, the action resulting from the previous step is invariant under the gauge-transformation

$$
\begin{aligned}
V_{\mu} & \rightarrow V_{\mu}+\nabla_{\mu} \zeta \\
\chi & \rightarrow \chi+M \zeta
\end{aligned}
$$


A useful choice for the gauge-condition is to associate the longitudinal component of $V$ with $\chi$. We now have a Faddeev-Popov scalar ghost and the resulting gaugefixed action is

$$
\begin{array}{r}
\tilde{S}=\int \mathrm{d}^{4} x \sqrt{g}\left[\frac{1}{2} \phi^{\mu \nu}\left(\Delta(1,1)-2 \Lambda+M^{2}\right) \phi_{\mu \nu}+V^{\mu}\left(\Delta\left(\frac{1}{2}, \frac{1}{2}\right)-2 \Lambda+M^{2}\right) V_{\mu}\right. \\
-\frac{1}{8}\left(\frac{-2 \Lambda+3 M^{2}}{-2 \Lambda+M^{2}}\right) \phi\left(\Delta(0,0)-2 \Lambda+M^{2}\right) \phi+\frac{-2 \Lambda+M^{2}}{M^{2}} \chi\left(\Delta(0,0)-2 \Lambda+M^{2}\right) \chi \\
+\frac{1}{2} \phi_{\mu \nu} j^{\mu \nu}+\frac{1}{8} \phi j
\end{array}
$$

for the massive case, and

$$
\begin{array}{r}
\tilde{S}=\int \mathrm{d}^{4} x \sqrt{g}\left[\frac{1}{2} \phi^{\mu \nu}\left(\Delta(1,1)-\frac{4}{3} \Lambda\right) \phi_{\mu \nu}+V^{\mu}\left(\Delta\left(\frac{1}{2}, \frac{1}{2}\right)-\frac{4}{3} \Lambda\right) V_{\mu}\right. \\
-2 \chi\left(\Delta(0,0)-\frac{4}{3} \Lambda\right) \chi+\frac{1}{2} \phi_{\mu \nu} j^{\mu \nu}+\frac{1}{8} \phi j
\end{array}
$$

for the partially massless case. The resulting one loop contributions $\Gamma^{(1)}[g]=-\ln Z[g, 0]$ are tabulated as

\begin{tabular}{|l|c|}
\hline$M^{2} \rightarrow 0$ & $M^{2}=0$ \\
\hline$-\frac{1}{2} \ln \operatorname{Det}\left(\Delta\left(\frac{1}{2}, \frac{1}{2}\right)-2 \Lambda+M^{2}\right)$ & $-\ln \operatorname{Det}\left(\Delta\left(\frac{1}{2}, \frac{1}{2}\right)-2 \Lambda\right)$ \\
$+\frac{1}{2} \ln \operatorname{Det}\left(\Delta(1,1)-2 \Lambda+M^{2}\right)$ & $+\frac{1}{2} \ln \operatorname{Det}(\Delta(1,1)-2 \Lambda)$ \\
& $+\frac{1}{2} \ln \operatorname{Det}(\Delta(0,0)-2 \Lambda)$ \\
\hline$M^{2} \rightarrow \frac{2}{3} \Lambda$ & $M^{2}=\frac{2}{3} \Lambda$ \\
\hline$-\frac{1}{2} \ln \operatorname{Det}\left(\Delta\left(\frac{1}{2}, \frac{1}{2}\right)-\frac{4}{3} \Lambda\right)$ & $-\frac{1}{2} \ln \operatorname{Det}\left(\Delta\left(\frac{1}{2}, \frac{1}{2}\right)-2 \Lambda+M^{2}\right)$ \\
$+\frac{1}{2} \ln \operatorname{Det}\left(\Delta(1,1)-\frac{4}{3} \Lambda\right)$ & $+\frac{1}{2} \ln \operatorname{Det}\left(\Delta(1,1)-2 \Lambda+M^{2}\right)$ \\
$-\frac{1}{2} \ln \operatorname{Det}\left(\Delta(0,0)-\frac{4}{3} \Lambda\right)$ & \\
\hline
\end{tabular}

It remains to check that there is no conspiracy among the eigenvalues of these operators that would make these expressions coincide. To show this, it suffices to calculate the coefficients in expansion for the graviton propagator associated with $S_{L}+S_{M}$, and compare it with the massless and the partially massless cases as can be determined from [7]. The trace of the heat kernel

$$
\operatorname{Tr} e^{-\Delta^{(\Lambda)} t}=\sum_{k=0}^{\infty} t^{(k-4) / 2} \int \mathrm{d}^{4} x \sqrt{g} b_{k}^{(\Lambda)}
$$

gives $b_{4}^{(\Lambda, M)}$ which determines the number of zero modes and is given by

\begin{tabular}{|l|c|}
\hline$M^{2} \rightarrow 0$ & $M^{2}=0$ \\
\hline$\left[b_{4}^{(\Lambda)}(1,1)-b_{4}^{(\Lambda)}\left(\frac{1}{2}, \frac{1}{2}\right)\right]$ & {$\left[b_{4}^{(\Lambda)}(1,1)-2 b_{4}^{(\Lambda)}(1 / 2,1 / 2)+b_{4}^{(\Lambda)}(0,0)\right]$} \\
$=200 R_{\mu \nu \rho \sigma} R^{\mu \nu \rho \sigma}-1740 \Lambda^{2}$ & $=212 R_{\mu \nu \rho \sigma} R^{\mu \nu \rho \sigma}-2088 \Lambda^{2}$ \\
\hline$M^{2} \rightarrow \frac{2}{3} \Lambda$ & $M^{2}=\frac{2}{3} \Lambda$ \\
\hline$\left[b_{4}^{(\Lambda)}(1,1)-b_{4}^{(\Lambda)}\left(\frac{1}{2}, \frac{1}{2}\right)\right]$ & {$\left[b_{4}^{(\Lambda)}(1,1)-b_{4}^{(\Lambda)}(1 / 2,1 / 2)-b_{4}^{(\Lambda)}(0,0)\right]$} \\
$=200 R_{\mu \nu \rho \sigma} R^{\mu \nu \rho \sigma}-740 \Lambda^{2}$ & $=199 R_{\mu \nu \rho \sigma} R^{\mu \nu \rho \sigma}-1096 \Lambda^{2}$ \\
\hline
\end{tabular}


We see that the full quantum theory for each of the massive and partially massless theories is discontinuous [6]. This is perhaps not surprising considering the different degrees of freedom in the three cases.

Recently the case of the gravitino has been studies classically for $\Lambda \neq 0$ [8] with conclusion similar to those in the case for the graviton. The quantum case is currently under investigation.

\section{ACKNOWLEDGEMENTS}

I would like to thank the organizers of the conference for invitation and for the interesting atmosphere. I also thank F. Dilkes, M.J. Duff and J.T. Liu for

collaboration on the subject of this talk and for discussions and suggestions. I also thank UM Rackham School for travel support.

\section{REFERENCES}

1. van Dam,H. and Veltman, M., Nucl. Phys. B22, 397-411 (1970); Zakharov, V. , JETP Lett. $12312-314$ (1970).

2. Higuchi, A., Nucl. Phys. B325, 745-765 (1989).

3. Porrati, M., Phys. Lett. 498B, 92-96 (2001); Kogan, I. Mouslopoulos, S. and Papazoglou, A., Phys. Lett. 503B, 173-180 (2001)

4. Deser, S. and Waldron, A., Phys. Rev. Lett. 87 031601, (2001).

5. Christensen, S.M. and Duff, M.J., Phys. Lett. 76B, 571-574 (1978).

6. Dilkes, F.A.,Duff, M.J., Liu, J.T. and Sati, H., Phys. Rev. Lett. 87041301 (2001); Duff, M.J., Liu, J.T. and Sati, H., Phys. Lett. 516B,156-160 (2001).

7. Christensen, S.M. and Duff, M.J., Nucl. Phys. B154, 301-342 (1979); ibid B170, 480-506 (1980).

8. Grassi, P. and van Nieuwenhuizen, P., Phys. Lett. 499B, 174-178 (2001); Deser, S. and Waldron, A., Phys.Lett. 501B, 134-139 (2001). 